\title{
Oscillation induced propagation of synchrony in structured neural networks
}

\author{
Sven Jahnke ${ }^{1,2,3^{*}}$, Raoul-Martin Memmesheimer ${ }^{4}$, Marc Timme ${ }^{1,2,3}$ \\ From Twenty Second Annual Computational Neuroscience Meeting: CNS*2013 \\ Paris, France. 13-18 July 2013
}

Spatio-temporally coordinated patterns of spiking activity have been experimentally observed in a range of neural circuits, but their dynamical origin is still not well understood. A prominent hypothesis states that propagating synchronized activity through embedded feed-forward networks might dynamically generate such patterns [1,2]. Modeling studies indeed showed that such "synfire-chains" embedded in random recurrent networks enable reliable signal transmission by propagating localized (sub-network) synchrony, if their structure is strongly pronounced compared to the embedding network. This requires in particular a dense connectivity between the neuronal layers of the chain or strongly enhanced synapses and modified response properties of neurons within the chain [3]. So far, however, such prominent large-scale structures have not been experimentally observed.

Single neuron experiments [4] indicate that neuronal dendrites are capable of nonlinearly amplifying sufficiently synchronous inputs by eliciting dendritic spikes, thereby inducing non-additive interactions. Here we demonstrate that such non-additive coupling promotes guided synchrony propagation already in random recurrent neural networks with mildly enhanced, biologically plausible sub-structures and without anatomically superimposed feed-forward chains [5]. Our analysis explains the mechanisms underlying robust propagation and shows in which sense non-additive enhancement - a local neuron property that dynamically changes with input synchrony - may complement dense and nonlocal structural connectivity.

Most neuronal circuits exhibit oscillations of various frequencies and amplitudes [6]. Such oscillations may

\footnotetext{
* Correspondence: sjahnke@nld.ds.mpg.de

${ }^{1}$ Network Dynamics, Max Planck Institute for Dynamics \& Self-Organization, Göttingen, Germany

Full list of author information is available at the end of the article
}

influence the dynamics of synchrony propagation. We thus further study this influence for both externally induced oscillations as well as for oscillations generated by the rhythmically propagating synchronous activity itself. We find that in networks with linear dendrites and balanced input, the oscillations hinder synchrony propagation, if they effect the dynamics at all. In contrast, for non-additive coupling, oscillations support synchronous propagation, if they are in resonance and the interplay between oscillations and propagating activity induces complex locking patterns: We show that in recurrent circuits containing high-connectivity (hub-)neurons, the oscillatory echo to propagating synchrony can generate synchrony in the remainder of the network and thereby in turn stabilize or even enable synchrony propagation along predefined paths: The network echo promotes signal transmission.

\section{Acknowledgements}

We acknowledge support by the BMBF (Grant No. 01GQ1005B) and the DFG (Grant No. TI 629/3-1).

\section{Author details}

'Network Dynamics, Max Planck Institute for Dynamics \& Self-Organization, Göttingen, Germany. ${ }^{2}$ Bernstein Center for Computational Neuroscience Göttingen, Göttingen, Germany. ${ }^{3}$ Fakultät für Physik, Georg-AugustUniversität Göttingen, Göttingen, Germany. ${ }^{4}$ Donders Institute, Department for Neuroinformatics, Radboud University, Nijmegen, Netherlands.

Published: 8 July 2013

\section{References}

1. Abeles M: Corticonics: Neuronal Circuits of the Cerebral Cortex Cambridge University Press; 1991.

2. Diesmann M, Gewaltig M-O, Aertsen A: Conditions for Stable Propagation of Synchronous Spiking in Cortical Neural Networks. Nature 1999, 402:529.

3. Kumar A, Rotter S, Aertsen A: Conditions for Propagating Synchronous Spiking and Asynchronous Firing Rates in a Cortical Network Model. J Neurosc 2008, 28:5268.

4. Vogels TP, Abbott LF: Signal Propagation in Networks of Integrate-andFire Neurons. J Neurosci 2005, 25:10786. 
5. Ariav G, Polsky A, Schiller J: Submillisecond precision of the input-output transformation function mediated by fast sodium dendritic spikes in basal dendrites of CA1 pyramidal neurons. J Neurosci 2003, 23:7750.

6. Gasparini S, Magee J: State dependent dendritic computation in hippocampal CA1 pyramidal neurons. J Neurosci 2006, 26:2088.

7. Losonczy A, Makara J, Magee J: Compartmentalized dendritic plasticity and input feature storage in neurons. Nature 2008, 452:4361.

8. Jahnke S, Timme M, Memmesheimer R-M: Guiding Synchrony through Random Networks. Phys Rev X 2012, 2:041016.

9. Memmesheimer R-M, Timme M: Non-Additive Coupling Enables Propagation of Synchronous Spiking Activity in Purely Random Networks. PLoS Comput Biol 2012, 8:e1002384

10. Buzsáki G, Draguhn A: Neuronal Oscillations in Cortical Networks. Science 2004, 304:1926.

doi:10.1186/1471-2202-14-S1-P390

Cite this article as: Jahnke et al:: Oscillation induced propagation of synchrony in structured neural networks. BMC Neuroscience 201314 (Suppl 1):P390.

\section{Submit your next manuscript to BioMed Central} and take full advantage of:

- Convenient online submission

- Thorough peer review

- No space constraints or color figure charges

- Immediate publication on acceptance

- Inclusion in PubMed, CAS, Scopus and Google Scholar

- Research which is freely available for redistribution

Submit your manuscript at www.biomedcentral.com/submit
Ciomed Central 